Next week, by a simple show of hands at an astronomy meeting, Earth could go from being one of nine planets to one of twelve - with unknown numbers yet to be discovered.

A seven-member panel appointed by the International Astronomical Union (IAU) has recommended a new definition of a planet: any body in orbit around a star that is not a star itself nor in orbit around a much larger planet, and that is massive enough for gravity to have squished it into an approximately spherical shape.

The IAU planned to introduce the notion in a draft resolution on 16 August, after Nature went to press, and will discuss it on 22 August during an open session at its general assembly in Prague, Czech Republic. Two days later, if a simple majority of IAU members vote for it or a slightly revised version, that will become the official scientific word on the topic.

Under the definition, most objects with a mass greater than $5 \times 10^{20}$ kilograms, and typically with a diameter greater than 800 kilometres, would qualify as planets. That would instantly elevate the asteroid Ceres and Pluto's moon Charon to planet status, as Ceres is roughly spherical and Pluto and Charon can

be described as a binary planet system. The Solar System's twelfth planet would be object $2003 \mathrm{UB}_{313}$, nicknamed Xena, one of many 'trans-neptunian objects' lurking on the Solar System's fringes.

Not all are happy with the 'roundness' criterion, however. "It's not what I think of when I think of a planet," says Michael Brown, an astronomer at the California Institute of Technology in Pasadena and co-discoverer of $\mathrm{UB}_{313}$. "I don't think of things that are a thousand times smaller than Earth."

The discovery of $\mathrm{UB}_{313}$ beyond Pluto's orbit last year brought matters to a head. Astronomers have estimated that it is at least 2,400 kilo-

\section{"If there is nothing} accepted, we have to sit in the silly place where we are now." metres in diameter - larger than Pluto, but smaller than any of the other eight planets.

All this demands a new definition of 'planet', some say. "If there is nothing accepted, we have to sit in the silly place where we are now: Pluto is a planet and $\mathrm{UB}_{313}$ is not," says Iwan Williams, an astronomer at Queen Mary, University of London.

Williams chaired an earlier IAU committee that was meant to define a planet, but it deadlocked last November. "People came in with well-defined ideas of what a planet should be, expressed them strongly, and hardly anybody

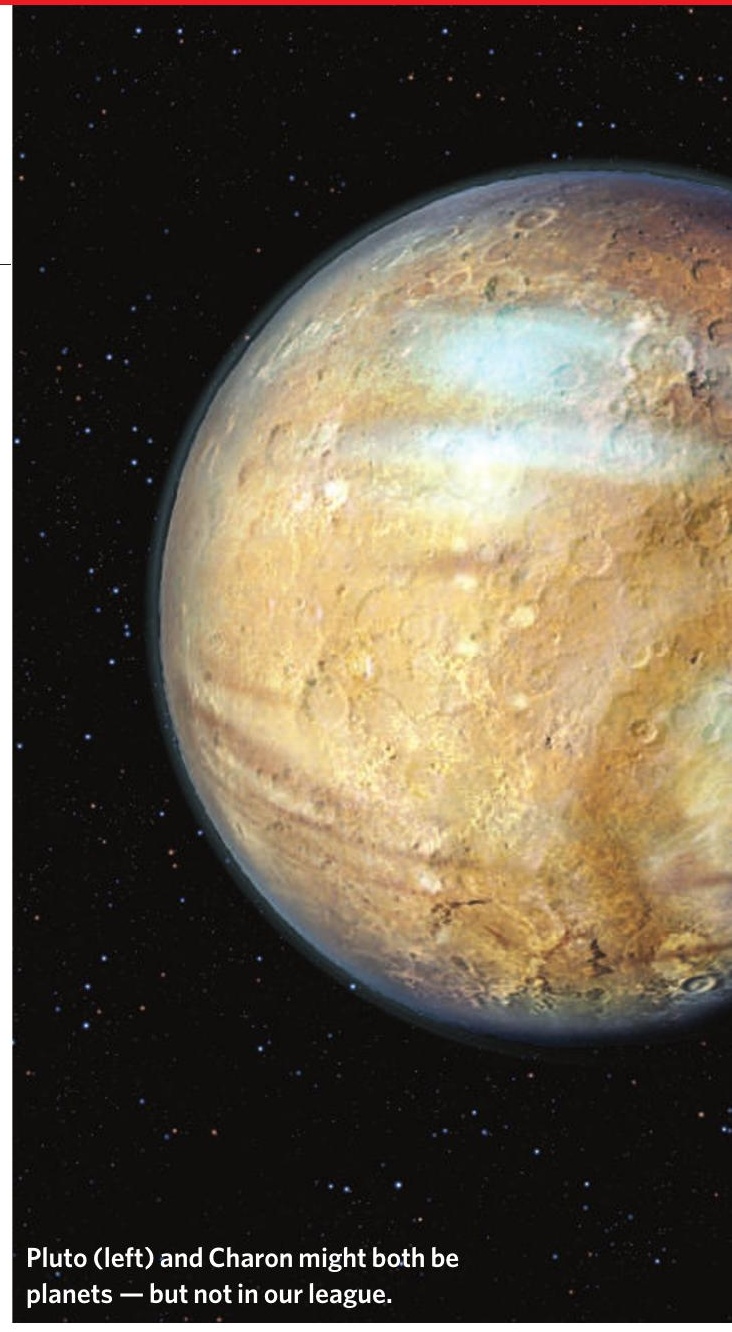

changed their mind," says committee member Alan Boss, an astronomer at the Carnegie Institute of Washington. Opinions were divided between three options to define a planet orbiting a star: any object with a diameter greater than 2,000 kilometres; any object massive enough for gravity to make it round; or any object that dominates its region of space.

Faced with that stalemate, the IAU appointed

\title{
AIDS meeting urged to rethink prevention strategy
}

\section{TORONTO}

Scientists, governments and funding agencies need to revise their strategy for deploying and testing HIV-prevention methods, delegates to the XVI International AIDS Conference in Toronto, Canada, heard this week.

Although conventional prevention methods, such as the use of condoms, have had some success, advocates say that women need to have more control over prevention. Various new approaches, including the externally applied gels known as microbicides or drugs taken orally, show promise, said leaders in the fight.
Challenges remain, however, in bringing such methods to the clinic. "There are serious obstacles that could significantly delay, or even derail, critical prevention trials - including inadequate resources and capacity to launch and complete trials, and emerging ethical concerns," warned a group of scientists, doctors and activists called the Global HIV Prevention Working Group.

A 15 August report from the coalition called on governments and scientists to focus on the most promising interventions, set up trial sites, and coordinate their plans to avoid wasting time and money duplicating efforts. It also advised the creation of a panel of experts that could provide ethical guidance for the trials. And it urged societies to prepare for the roll-out of new prevention methods if they prove successful.

The warnings aim to reduce the problems already hampering efforts to find alternative prevention methods. For example, activists' concerns, such as over whether people who contracted HIV during the trial would receive treatment afterwards, shut down two trials on preventive drugs in Cambodia and Cameroon in 2004 and 2005.

Scientists hope that interest from big funding groups, such as the Bill and Melinda Gates Foundation, based in Seattle, Washington, could focus attention on these preventive efforts.

Trials are currently testing the effects on HIV infection of microbicides, orally-ingested drugs, male circumcision, barriers such as diaphragms and the treatment of herpes infections. But the studies are costly and difficult to run.

"We need to make strategic choices, and I hope Gates is going to give leadership in the way that is finally happening in the vaccine field," says Joep Lange, chairman of the International AIDS Society's 
